\title{
Improved stellar parameters of CoRoT-7 ${ }^{\star}$ A star hosting two super Earths
}

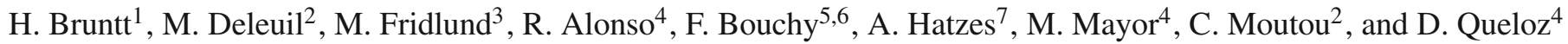 \\ 1 LESIA, Observatoire de Paris-Meudon, 5 place Jules Janssen, 92195 Meudon, France \\ e-mail: bruntt@phys.au.dk \\ 2 LAM, UMR 6110, CNRS/Université de Provence, 38 rue F. Joliot-Curie, 13388 Marseille, France \\ 3 ESA, ESTEC, SRE-SA, Keplerlaan 1, 2200AG, Noordwijk, The Netherlands \\ 4 Observatoire de Genève, Université de Genève, 51 Ch. des Maillettes, 1290 Sauverny, Switzerland \\ 5 Institut d'Astrophysique de Paris, UMR7095 CNRS, Université Pierre \& Marie Curie, 98bis Bd Arago, 75014 Paris, France \\ 6 Observatoire de Haute-Provence, CNRS/OAMP, 04870 St Michel l'Observatoire, France \\ 7 Thüringer Landessternwarte Tautenburg, Sternwarte 5, 07778 Tautenburg, Germany
}

Received 27 January 2010 / Accepted 17 May 2010

\section{ABSTRACT}

\begin{abstract}
Context. Accurate parameters of the host stars of exoplanets are needed for interpreting the new planetary systems. The CoRoT satellite recently discovered a transiting rocky planet with a density similar to the inner planets in our solar system, a so-called super Earth. The mass was determined using ground-based follow-up spectroscopy, which also revealed a second, non-transiting super Earth.

Aims. These planets are orbiting a relatively faint $\left(m_{V}=11.7\right)$ G9V star called CoRoT-7. We wish to refine the determination of the physical properties of the host star, which are important for the interpretation of the properties of the planetary system.

Methods. We used high-quality spectra from HARPS at the ESO $3.6 \mathrm{~m}$ and UVES at the VLT $8.2 \mathrm{~m}$. We used various methods to analyse the spectra using 1D LTE atmospheric models. From the analysis of Fe I and Fe II lines we determined the effective temperature, surface gravity and microturbulence. We used the Balmer lines to constrain the effective temperature and pressuresensitive $\mathrm{Mg} 1 \mathrm{~b}$ and $\mathrm{Ca}$ lines to constrain the surface gravity. We analysed both single spectra and co-add spectra to identify systematic errors. We determine the projected rotational velocity and macroturbulence by fitting the line shapes of isolated lines. We finally determined an approximate absolute magnitude from the Wilson-Bappu effect.

Results. From the analysis of the three best spectra, we find $T_{\text {eff }}=5250 \pm 60 \mathrm{~K}, \log g=4.47 \pm 0.05,[\mathrm{M} / \mathrm{H}]=+0.12 \pm 0.06$, and $v \sin i=1.1_{-0.5}^{+1.0} \mathrm{~km} \mathrm{~s}^{-1}$. The chemical composition of 20 analysed elements is consistent with uniform scaling by the metallicity +0.12 dex. From the analysis of spectra of stars with well-known parameters with similar parameters to CoRoT-7 (the Sun and $\alpha$ Cen B) we demonstrate that our methods are robust within the claimed uncertainties. We compared the $L / M$ ratio with isochrones to constrain the evolutionary status. Using the age estimate of 1.2-2.3 Gyr based on stellar activity, we determine the mass and radius $0.91 \pm 0.03 M_{\odot}$ and $0.82 \pm 0.04 R_{\odot}$. With these updated constraints we fitted the CoRoT transit light curve for CoRoT-7b. The revised planet radius is slightly smaller, $R=1.58 \pm 0.10 R_{\oplus}$, and the density becomes slightly higher, $\rho=7.2 \pm 1.8 \mathrm{~g} \mathrm{~cm}^{-3}$.
\end{abstract}

Key words. stars: abundances - stars: late-type - stars: fundamental parameters

\section{Introduction}

The discovery of the first super Earth planet outside of the Solar system with a measured absolute mass and radius has recently been announced, and was based on photometric data from CoRoT (Convection, Rotation and planetary Transits; Baglin et al. 2006). This planet, CoRoT-7b, has a radius of $1.68 \pm 0.09 R_{\oplus}$ (Léger et al. 2009, hereafter LRS09), mass $4.8 \pm 0.8 M_{\oplus}$ (Queloz et al. 2009), and the orbital period is about 0.85 days (LRS09). The average density is $5.6 \pm 1.3 \mathrm{~g} \mathrm{~cm}^{-3}$ which is similar to Mercury, Venus, and the Earth (Queloz et al. 2009). Furthermore, a second non-transiting super Earth has been found from radial-velocity monitoring (Queloz et al. 2009). These results have only been possible thanks to an extensive ground-based follow-up programme of the relatively faint star

* The CoRoT space mission, launched on December 27, 2006, has been developed and is being operated by CNES, with the contribution of Austria, Belgium, Brazil, ESA, The Research and Scientific Support Department of ESA, Germany and Spain.
CoRoT-7 (TYCHO ID 4799-1733-1; $m_{V}=11.7$ ) over more than one year.

In the derivation of the planetary parameters, one of the most important factors is correct identification of the host star's fundamental parameters and evolutionary stage. It is particularly important to estimate the stellar radius, which is imperative for determining the absolute planetary radius. A first analysis by several of the CoRoT teams has been carried out in LRS09, based on a spectrum from the "Ultraviolet and Visual Echelle Spectrograph" (UVES@VLT $8.2 \mathrm{~m}$ ) and a preliminary analysis of 53 co-added spectra from the "High Accuracy Radial velocity Planet Searcher" (HARPS@ESO 3.6 m).

Since then, a total of 107 spectra from HARPS have become available (Queloz et al. 2009). These spectra have higher spectral resolution and better signal-to-noise $(S / N)$ than the UVES spectrum analysed by LRS09. We can therefore now refine the analysis of CoRoT-7 and possibly impose stronger constraints on the properties of the system. The methods we employ have been developed during the analysis of other CoRoT targets 
Table 1. The 10 spectra used for the spectroscopic analysis.

\begin{tabular}{lcccr}
\hline \hline Spec. & Date & Time & $t_{\exp }$ & \\
ID & UT & UT & [s] & \multicolumn{1}{c}{$S / N$} \\
\hline H1 & $2008-12-26$ & $04: 56$ & 2700 & 95 \\
H2 & $2009-01-15$ & $05: 39$ & 2700 & 90 \\
H3 & $2009-01-17$ & $01: 45$ & 2700 & 95 \\
H1-7 & Combined spec. & & & 235 \\
H1-107 & Combined spec. & & & 700 \\
U1 & $2008-09-13$ & $08: 39$ & 3600 & 470 \\
\hline Ceres & $2006-07-16$ & $07: 50$ & 1800 & 220 \\
Ganymede & $2007-04-13$ & $09: 40$ & 300 & 340 \\
Moon & $2008-08-09$ & $02: 39$ & 300 & 400 \\
\hline$\alpha$ Cen B & Combined spec. & & 1030 \\
\hline \multicolumn{7}{l}{}
\end{tabular}

Notes. $\mathrm{H} 1$ to $\mathrm{H} 3$ are individual HARPS spectra, $\mathrm{H} 1-7$ is 7 co-added spectra, and H1-107 is the weighed sum of 107 spectra. U1 is the UVES spectrum. Ceres, Ganymede, and Moon are solar spectra from HARPS, while $\alpha$ Cen B refers to 25 co-added HARPS spectra from 2004-05-15.

(Deleuil et al. 2008; Moutou et al. 2008; Rauer et al. 2009; Fridlund et al. 2010; Bruntt 2009). In the current paper we have expanded these tools and describe our approach in greater detail than previously. These tools will be the standard methods to apply for characterising future CoRoT targets.

\section{Spectroscopic observations}

We initially acquired one UVES spectrum confirming that the star is a dwarf, meaning the absolute radius of the planet must be small (LRS09). To constrain the mass of CoRoT-7b, a series of 107 spectra were collected with the HARPS spectrograph between March 2008 and February 2009 (Queloz et al. 2009). The HARPS spectrograph has a spectral resolution of 115000 (Mayor et al. 2003), covering the optical range from $3827 \AA$ to $6911 \AA$. With exposure times of 1800 or 2700 s, the $S / N$ of the individual spectra varies from $\simeq 30-90$, depending on the conditions at the time of observation.

We used the data from the standard HARPS pipeline, and each order was divided by the blaze function to get an approximately rectified spectrum. We shifted each spectrum by the radial velocity to set it to the heliocentric rest frame, using the values from Queloz et al. (2009). Each spectrum was rebinned to the same wavelength grid with a constant step of $0.01 \AA$.

We suspected that some of the exposures could be affected by reflected moonlight. While this data can be used for measuring of the radial velocity variation, scattered light can potentially affect the relative line depths and hence systematically affect the analysis. In order to identify such potential systematic errors, we made different combinations of the spectra as presented in Table 1 . We selected seven spectra acquired during dark time, and with the highest $S / N$, computed in nearly linefree regions around $5830 \AA$. The co-addition, order per order, of these 7 spectra gives the H1-7 combined spectrum. We also analysed three individual HARPS spectra with the highest $S / N$ (H1, H2, and H3). We finally co-added all HARPS spectra using weights $w \propto S / N$ to get the H1-107 spectrum.

A preliminary analysis of the UVES spectrum of CoRoT-7 was described in LRS09. This spectrum has a lower resolution $(R=65000)$ than HARPS. We include our updated analysis here for completion.

To calibrate our methods, we analysed three HARPS spectra of the Sun, available from the ESO/HARPS intrumentation website $^{1}$. The spectra were acquired by observing Ceres, Ganymede and the Moon, and have $S / N$ around 250, 350, and 450 , respectively. The "Moon" solar spectrum was observed in the high-efficiency EGGS mode, which has resolution $R=$ 80000 . In addition, we analysed a co-added HARPS spectrum of $\alpha$ Cen B, which has similar parameters to CoRoT-7. $\alpha$ Cen B has been studied using direct, model-independent methods (interferometry, binary orbit), so its absolute parameters (mass, radius, luminosity and $T_{\text {eff }}$ ) are known with high accuracy (Porto de Mello et al. 2008). We use this to evaluate our indirect methods that rely on the validity of the spectral analysis using 1D LTE atmospheres.

\section{Versatile wavelength analysis (VWA)}

We used the VWA package (Bruntt et al. 2004, 2008, 2010; Bruntt 2009) to analyse the spectra listed in Table 1 . It can perform several tasks from normalisation of the spectrum, selection of isolated lines for abundance analysis, iterative fitting of atmospheric parameters, and determination of the projected rotational velocity $(v \sin i)$. The basic tools of VWA have been described in previous work (Bruntt et al. 2002), and here we give a more complete description of some additional tools in relation to the results we determine for CoRoT-7.

\subsection{Normalisation of the spectra}

In Fig. 1 we illustrate the principles of the rainbow program that we used to normalise the spectra. The top panels show a wide wavelength range in a single order, and the bottom panels show a zoom near the edge of the same echelle order. The user must manually identify continuum points by comparing the observed spectrum with a template, which is usually a synthetic spectrum calculated with the same approximate parameters as the star. The top panel in Fig. 1 shows the spectrum before normalisation where eight continuum points have been identified. A spline function - optionally a low-order polynomial - is fitted through these points. The spectrum from the adjacent echelle order is shown. The lower panel shows the normalised spectrum along with the template spectrum. The agreement with the adjacent order is very good, and there is acceptable agreement with the template. The overlapping orders are finally merged to improve the $S / N$ by up to $40 \%$.

When the continuum points have been marked for all orders, the normalised spectrum is saved. When the first spectrum has been normalised, the continuum points are re-used for the other spectra. We then carefully check the normalisation in each case since several readjustments are needed, especially in the blue end of the spectrum.

The high $S / N$ in the spectrum shown in Fig. 1 would indicate that the continuum is determined to better than $0.5 \%$. This is only true if the adopted synthetic template spectrum is identical to that of the star, i.e. the atomic line list is complete and the temperature and pressure structure of the atmosphere model represents the real star. From comparison of the template and normalised spectra in several regions (as in Fig. 1), we estimate that the continuum is correct to about $0.5 \%$, while discrepancies of 1-2\% may occur in regions where the degree of blending is high, and in the region of the wide Balmer lines and the $\mathrm{Mg} \mathrm{Ib}$ lines.

\footnotetext{
1 URL: http://wWW . eso.org/sci/facilities/lasilla/ instruments/harps/inst/monitoring/sun.html
} 

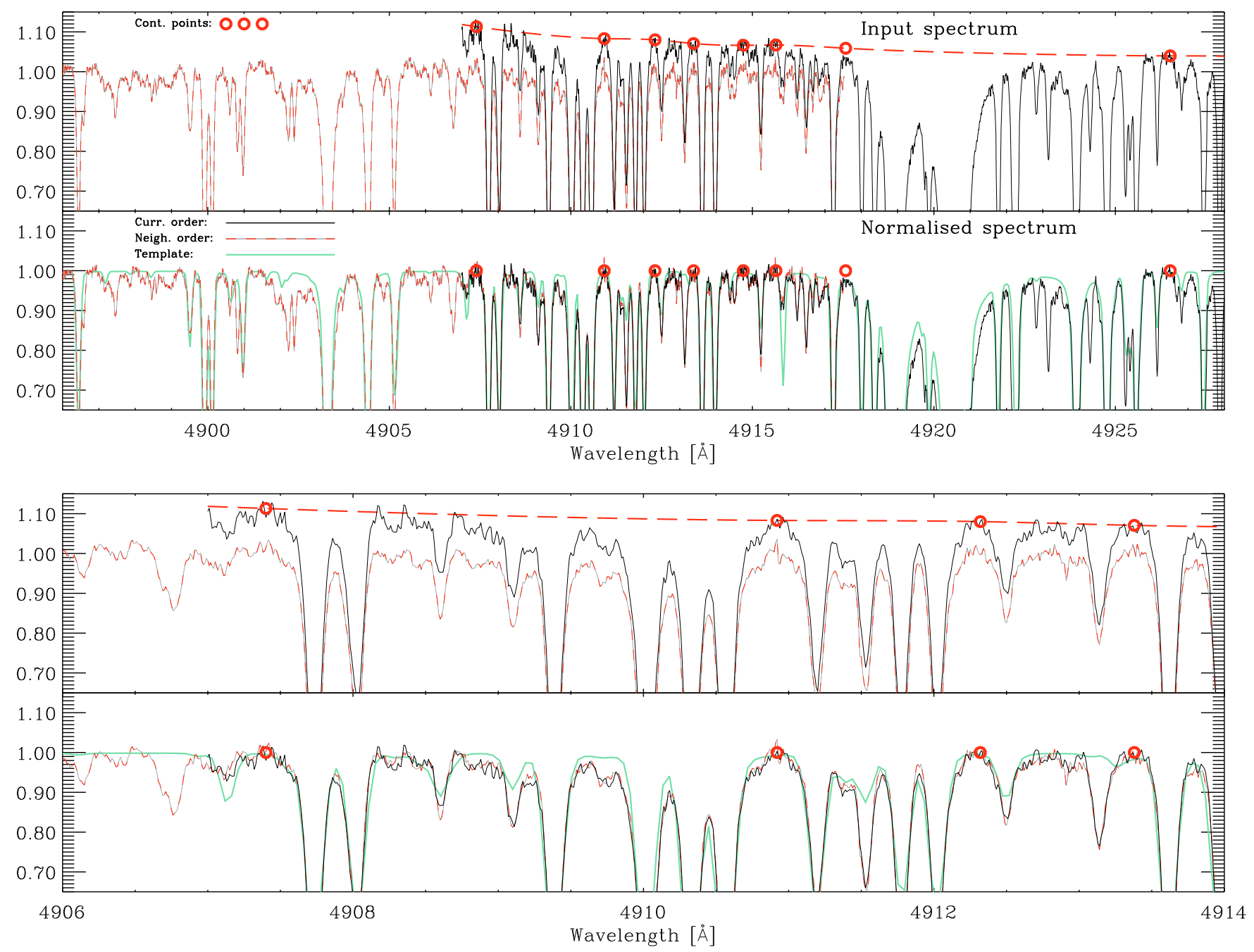

Fig. 1. A section of the CoRoT 7/H1-7 spectrum illustrating how the spectrum is normalised with rainbow. The top panels show a wide range and the lower panels show a zoom near the edge of the same echelle order. The neighbouring order is shown with a short-dashed line. The thick long-dashed line is a spline fit to the continuum windows marked by circles. The normalised spectrum agrees reasonably well with the template synthetic spectrum (smooth green line). The agreement between the two overlapping orders is good and will finally be merged to improve the $S / N$.

\subsection{Determination of $T_{\text {eff }}$ and $\log g$ from $\mathrm{Fe}_{\mathrm{I}-\mathrm{II}}$ lines}

This part of the VWA program has been described in some detail by Bruntt et al. (2002) and we will specify some updated details here. VWA uses 1D LTE atmosphere models interpolated in grids from MARCS (Gustafsson et al. 2008) or modified ATLAS9 models (Heiter et al. 2002). We adopted MARCS models for this study. The atomic line data we extracted from VALD (Kupka et al. 1999), which is a collection from many different sources. The synthetic profiles we computed with synth (Valenti \& Piskunov 1996). The VWA abundances we measured differentially with respect to a solar spectrum. We used the FTS spectrum by Kurucz et al. (1984), which was published in electronic form by Hinkle et al. (2000). We found that making a differential abundance analysis significantly improves the precision on the determined $T_{\text {eff }}$ and $\log g$ (see Bruntt et al. 2008). We assess the question of accuracy in Sects. 3.4 and 6.1.

VWA consists of three main programs written in IDL. Each program has a graphical user interface, called vwaview, vwaexam, and vwares. In vwaview the user can inspect the observed spectrum and select a set of isolated lines. They are fitted iteratively by computing synthetic profiles and adjusting the abundance until the observed and synthetic profiles have identical equivalent widths within a fixed wavelength range. The program vwaexam is used to inspect how well the synthetic profiles fit the observed lines. The user can manually reject lines or base the rejection on objective criteria such as reduced $\chi^{2}$ values and the relative line depths. It takes about one hour to fit 500 lines on a modern laptop. This is done with the program vwatask for fixed values of $T_{\text {eff }}, \log g$ and $v_{\text {micro }}$. The process is then repeated with various values of these parameters to measure the sensitivity of each line. The user can then investigate this sensitivity in the program vwares.

In Fig. 2 we show an example from vwares using the H1-7 spectrum of CoRoT-7. The abundances from Fe I and Fe II lines are plotted versus equivalent width ( $E W$; left panels) and excitation potential (EP; right panels) for four different sets of atmospheric parameters. The top panels are for the preferred parameters where we have minimised the correlation of Fe I with both $E W$ and EP and the mean abundances of Fe I and Fe II agree. The second panel is for $T_{\text {eff }}$ decreased by $300 \mathrm{~K}$, resulting in a clear correlation with $\mathrm{EP}$, and $\mathrm{Fe} \mathrm{I}$ and $\mathrm{Fe}$ II no longer agree. For the third panel, $\log g$ was decreased by 0.3 dex, leading to a low mean abundance of Fe II. The bottom panel is for 
Table 2. Determined atmospheric parameters for CoRoT-7, the Sun, and $\alpha$ Cen B.

\begin{tabular}{|c|c|c|c|c|c|c|c|c|c|c|}
\hline \multirow[b]{2}{*}{ Spec. } & \multicolumn{4}{|c|}{$\overline{\langle\text { Fe I-II }\rangle}$} & \multirow{2}{*}{$\begin{array}{r}\mathrm{Mg} 1 \mathrm{~b}\rangle \\
\log g\end{array}$} & \multirow{2}{*}{$\begin{array}{c}\langle\text { Ca6122 } \\
\log g\end{array}$} & \multirow{2}{*}{$\begin{array}{c}\langle\mathrm{Ca} 6162\rangle \\
\log g\end{array}$} & \multirow{2}{*}{$\begin{array}{c}\langle\mathrm{Ca} 6439\rangle \\
\log g\end{array}$} & \multicolumn{2}{|c|}{$\langle$ 〈Isol. lines $\rangle$} \\
\hline & $T_{\text {eff }}[\mathrm{K}]$ & $\log g$ & {$[\mathrm{Fe} / \mathrm{H}]$} & $v_{\text {micro }}\left[\mathrm{km} \mathrm{s}^{-1}\right]$ & & & & & $v \sin i$ & $v_{\text {macro }}$ \\
\hline H1 & $5180 \pm 67$ & $4.30 \pm 0.08$ & $+0.11 \pm 0.13$ & $0.98 \pm 0.07$ & $3.89 \pm 0.46$ & $4.44 \pm 0.08$ & $4.43 \pm 0.10$ & $4.34 \pm 0.19$ & 1.3 & 1.2 \\
\hline H1 SME & $5280 \pm 44$ & $4.44 \pm 0.06$ & $+0.13 \pm 0.06$ & $80 \pm($ & 4.62 & 4.66 & 4.53 & & & \\
\hline $\mathrm{H} 2$ & $00 \pm 41$ & $4.46 \pm 0.08$ & $+0.14 \pm 0.13$ & 0.09 & $4.02 \pm 0.47$ & $4.43 \pm 0.05$ & $4.56 \pm 0.06$ & $4.13 \pm 0.35$ & 1.1 & 1.2 \\
\hline H3 & $350 \pm 31$ & $4.57 \pm 0.06$ & $+0.15 \pm 0.09$ & $0.76 \pm 0.07$ & $4.14 \pm 0.44$ & $4.50 \pm 0.09$ & $4.77 \pm 0.07$ & $4.72 \pm 0.26$ & 1.1 & 1.2 \\
\hline H1-7 & $5280 \pm 35$ & $4.48 \pm 0.06$ & $+0.13 \pm 0.09$ & $0.80 \pm 0.05$ & $3.94 \pm 0.52$ & $4.47 \pm 0.06$ & $4.60 \pm 0.08$ & $4.32 \pm 0.27$ & 0.9 & 1.4 \\
\hline H1-107 & $5300 \pm 25$ & $4.54 \pm 0.05$ & $+0.13 \pm 0.08$ & $0.77 \pm 0.05$ & $3.91 \pm 0.55$ & $4.51 \pm 0.06$ & $4.58 \pm 0.05$ & $4.45 \pm 0.18$ & & \\
\hline H1-107 SME & $5290 \pm 44$ & $4.49 \pm 0.06$ & $+0.13 \pm 0.06$ & $0.80 \pm 0.05$ & 4.43 & 4.49 & 4.49 & & & \\
\hline U1 & $5300 \pm 17$ & $4.50 \pm 0.03$ & $+0.11 \pm 0.06$ & $.70 \pm 0.08$ & $3.94 \pm 0.49$ & $4.42 \pm 0.05$ & $4.46 \pm 0.06$ & $4.41 \pm 0.19$ & & \\
\hline Ceres & $5767 \pm 17$ & $4.44 \pm 0.03$ & $-0.01 \pm 0.03$ & $1.01 \pm 0.03$ & $4.50 \pm 0.08$ & $4.46 \pm 0.20$ & $4.43 \pm 0.10$ & $4.43 \pm 0.42$ & 1.4 & 2.1 \\
\hline Ganymede & $5757 \pm 17$ & $4.43 \pm 0.04$ & $-0.00 \pm 0.04$ & $0.93 \pm 0.03$ & $4.51 \pm 0.10$ & $4.33 \pm 0.17$ & $4.38 \pm 0.08$ & $4.47 \pm 0.37$ & 1.1 & 2.3 \\
\hline Moon & $775 \pm 25$ & $4.48 \pm 0.03$ & $+0.02 \pm 0.04$ & $0.91 \pm 0.04$ & $4.41 \pm 0.08$ & $4.53 \pm 0.20$ & $4.41 \pm 0.14$ & $4.50 \pm 0.33$ & 2.1 & 2.2 \\
\hline$\alpha$ Cen B & $5185 \pm 25$ & $4.50 \pm 0.03$ & $+0.31 \pm 0.05$ & $0.83 \pm 0.04$ & $4.01 \pm 0.50$ & $4.53 \pm 0.07$ & $4.51 \pm 0.05$ & $4.65 \pm 0.16$ & 1.0 & 0.8 \\
\hline
\end{tabular}

Notes. Results are from VWA except H1 and H1-107, which are also given for the SME analysis. The 1- $\sigma$ uncertainties are internal errors.

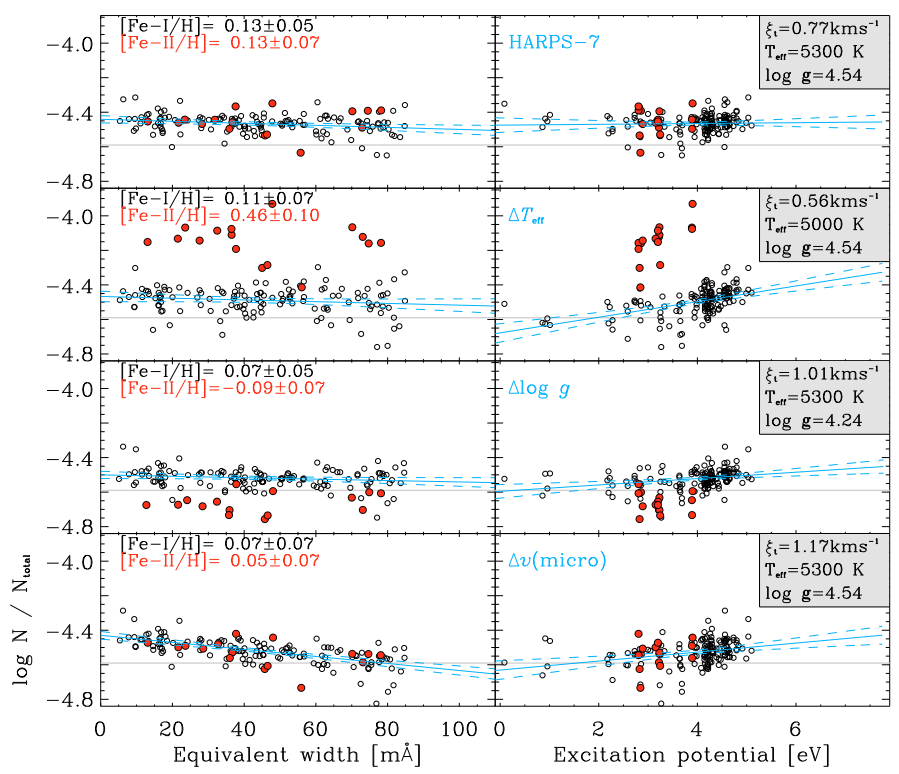

Fig. 2. Abundances of Fe I and Fe II shown as open and solid red circles, respectively, and plotted versus equivalent width and excitation potential (plot from the vwares program). The abundances are from the analysis of the H1-7 spectrum for four different sets of atmospheric models. The top panel is for the preferred model, the second panel is for a lower $T_{\text {eff }}$, the third panel for lower $\log g$, and the bottom panel is for higher $v_{\text {micro }}$. Also indicated is the solar Fe abundances (thin horizontal line) and a linear fit with $95 \%$ confidence limits indicated by the solid and dashed lines.

microturbulence increased by $0.4 \mathrm{~km} \mathrm{~s}^{-1}$, which leads to correlations of $\mathrm{Fe}$ I with both $E W$ and EP. From such analyses we can determine the "internal" uncertainty on the atmospheric parameters (see Bruntt et al. 2008, for a discussion).

In Fig. 3 we show an example of the abundances of six elements determined for the $\mathrm{H} 1-107$ spectrum. The mean abundance and rms error is given in the right panels. While Fe has the most lines, $\mathrm{Ti}, \mathrm{Cr}$, and $\mathrm{Ni}$ also show no strong correlation with equivalent with or excitation potential, indicating that the atmospheric model parameters are correct.

The atmospheric parameters for the six spectra of CoRoT-7 are summarised in Table 2. The applied method is indicated in angled brackets in the first row. There is good agreement between these results, although the $\mathrm{H} 1$ spectrum gives a systematically lower $T_{\text {eff }}$ and $\log g$. This is due to the correlation

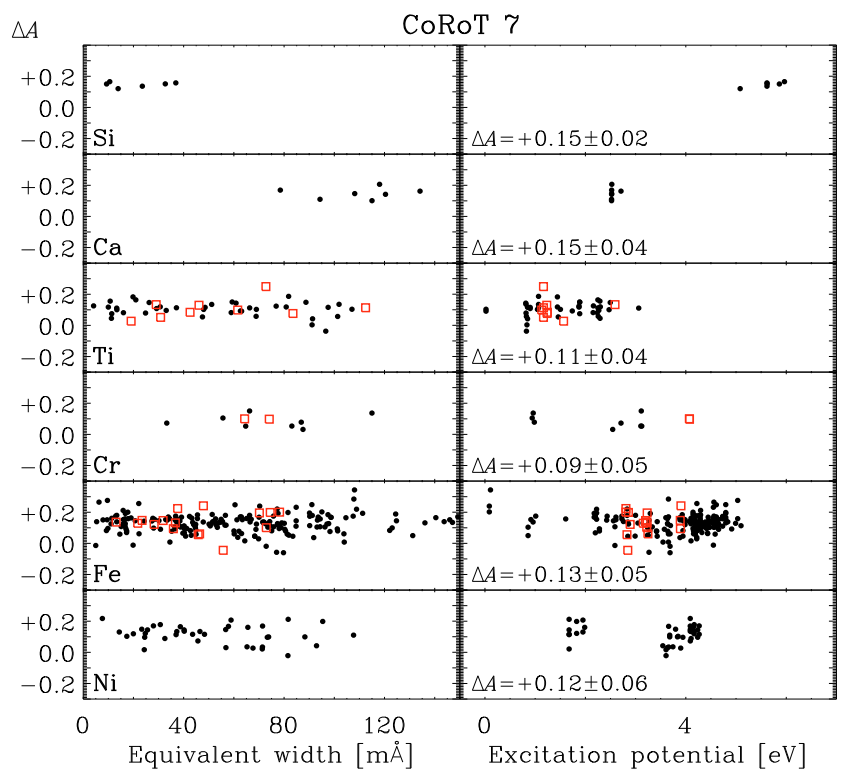

Fig. 3. Abundances determined from the H-107 spectrum for six elements plotted versus equivalent width and excitation potential. Solid and open symbols are used for neutral and ionised Fe lines, respectively.

between the two parameters as also noted by Bruntt (2009). He proposed that this degeneracy could be a problem for spectra with relatively low $S / N$ (H1 has $S / N=57$ ). For our final value of $T_{\text {eff }}$ and $\log g$ of CoRoT-7, we adopt the weighted mean value of the analysis of the three composite spectra: H17, H1-107, and U1: $T_{\text {eff }}=5297 \pm 13 \mathrm{~K}, \log g=4.51 \pm 0.02$, $v_{\text {micro }}=0.77 \pm 0.03 \mathrm{~km} \mathrm{~s}^{-1}$. The uncertainties stated here are internal errors. We assess the question of "realistic uncertainties" in Sect. 6.

\subsection{Determination of $\log g$ from wide lines}

The surface gravity of late-type stars can be determined from the Mg I b lines, the $\mathrm{NaID}$, and the Ca lines at $\lambda 6122, \lambda 6162$, and $\lambda 6439 \AA$. For Mg I b we used only the line at $\lambda 5184 \AA$ because the two lines around $\lambda 5170 \AA$ are too blended. We followed the approach of Fuhrmann et al. (1997) to adjust the van der Waals constants (pressure broadening due to hydrogen collisions) by requiring that our reference spectrum of the Sun (Hinkle et al. 2000) produces the solar value $\log g=4.437$. In Table 3 we 

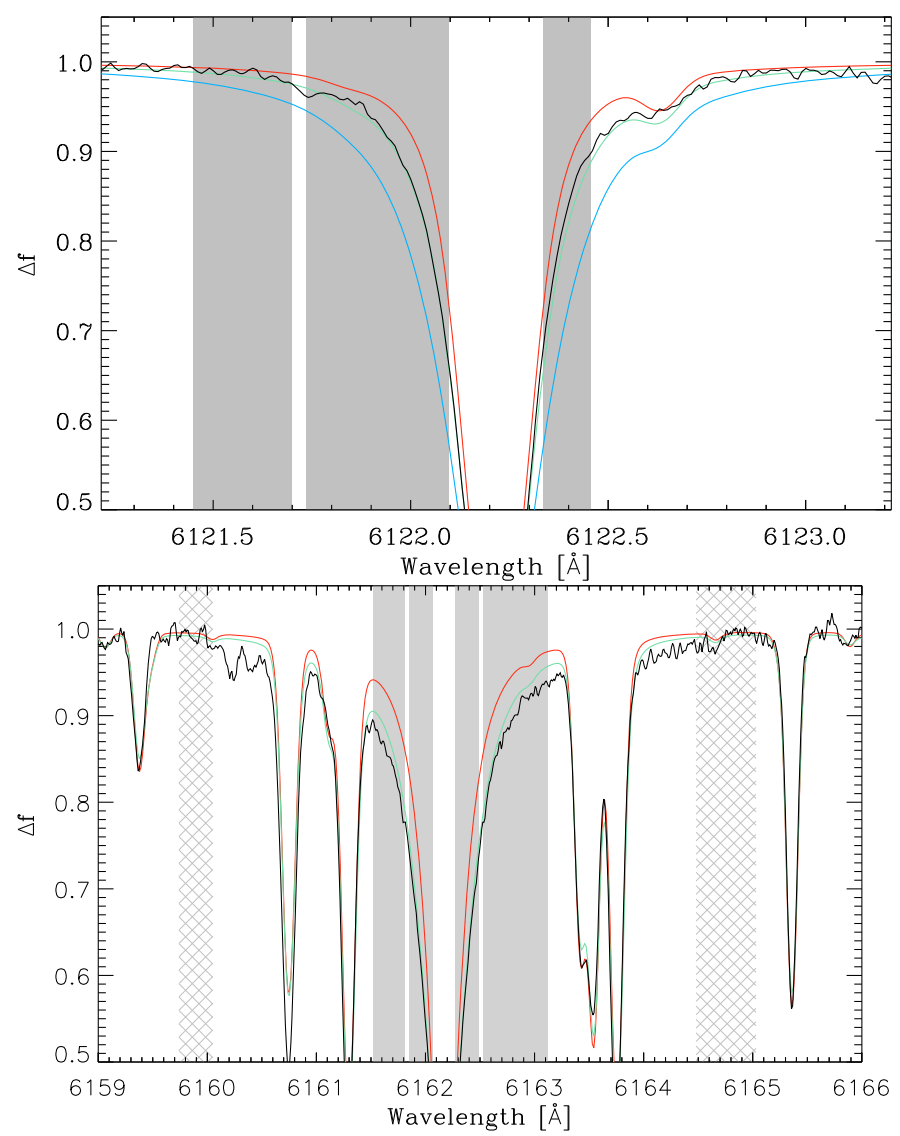

Fig. 4. The sensitivity of synthetic Ca lines fitted to the observed spectra of the Sun (top panel; Ganymede spectrum) and CoRoT-7 (bottom panel; H1-7 spectrum). The rectangles mark the areas used to compute the reduced $\chi^{2}$ and the hatched regions are used to normalise the spectrum.

list the adjusted van der Waals parameters along with the values extracted from VALD (from Barklem et al. 2000). Following the convention of VALD, it is expressed as the logarithm (base 10) of the full-width half-maximum per perturber number density at $10000 \mathrm{~K}$. The abundances of the fitted lines are determined from weak lines with $E W<120 \mathrm{~m} \AA$. The broadening due to $v \sin i$ and $v_{\text {macro }}$ is determined as described in Sect. 3.6. Examples of fitting the $\lambda 6122 \AA$ and $\lambda 6162 \AA$ lines are shown in Fig. 4 for the Sun (top panel) and CoRoT-7 (bottom panel). The synthetic profiles computed for the Sun have $\log g=4.04,4.44$ and 4.84. For the CoRoT-7 spectrum synthetic profiles have $\log g=4.08$ and 4.48. In each case a higher $\log g$ means the synthetic line becomes wider. The hatched regions are used to renormalise the spectrum by a linear fit. The rectangles mark regions where reduced $\chi^{2}$ values are computed, and they are used to determine the best value of $\log g$ and the $1-\sigma$ uncertainty.

We found that the MgIb line in CoRoT-7 is not very sensitive and gave lower values $(\log g \approx 4.0 \pm 0.5)$ than the Ca lines $(\log g \approx 4.5 \pm 0.1)$. The reason may be the high degree of blending with weaker lines for such a late type star. Since the higher value of $\log g$ is in good agreement with the result using Fe I-II, we neglect the results for the $\mathrm{Mg}$ I b lines. There is good agreement for the $\log g$ from the individual spectra. For the value of $\log g$ we adopt the weighted mean of the three composite spectra: $\log g=4.50 \pm 0.02$. The stated error does not include systematic errors, see Sects. 3.4 and 6.1.
Table 3. The adjusted Van der Waals constants compared to the values from VALD.

\begin{tabular}{lccc}
\hline \hline & & \multicolumn{2}{c}{$\log \gamma\left[\mathrm{rad} \mathrm{cm}^{3} / \mathrm{s}\right]$} \\
Line & $\lambda[\AA]$ & Adjusted & VALD \\
\hline Mg Ib & 5167.321 & -7.42 & -7.267 \\
& 5172.684 & -7.42 & -7.267 \\
& 5183.604 & -7.42 & -7.267 \\
\hline Na ID & 5889.951 & -7.85 & -7.526 \\
& 5895.924 & -7.85 & -7.527 \\
\hline Ca I & 6122.217 & -7.27 & -7.189 \\
& 6162.173 & -7.27 & -7.189 \\
\hline Ca I & 6439.075 & -7.84 & -7.569 \\
\hline
\end{tabular}

\subsection{Results for the Sun and $\alpha$ Cen B}

It is important to confirm that the employed spectroscopic methods produce trustworthy results. We therefore analysed two fundamental stars for which $T_{\text {eff }}$ and $\log g$ are known with very high accuracy: the Sun and $\alpha$ Cen $\mathrm{B}$. We analysed three single HARPS spectra of the Sun and one co-added spectrum of $\alpha$ Cen B. The results are summarised in Table 2 .

The parameters from the three solar spectra agree very well with the solar values. The canonical value for $T_{\text {eff }}$ is $5777 \mathrm{~K}$ (Cox 2000), and $\log g$ calculated from the Solar mass and radius is 4.437. The largest deviation is $20 \mathrm{~K}$ for $T_{\text {eff }}$ based on the analysis of Fe I-II lines. The surface gravity is constrained by several methods (Fe I-II, Mg I, Ca lines), but the largest deviation from the canonical value is only 0.1 dex. From Table 2 it is seen that some lines are less useful for constraining $\log g$ : Ca $\lambda 6439 \AA$ is the least sensitive line. For the weighted average, using the $\mathrm{Mg}$ and three $\mathrm{Ca}$ lines, we find excellent agreement for the three Solar HARPS spectra: $\log g=4.47 \pm 0.06,4.42 \pm 0.06$, and $4.43 \pm 0.06$.

For $\alpha$ Cen B we find $T_{\text {eff }}=5185 \pm 25 \mathrm{~K}, \log g=4.50 \pm 0.03$, and $[\mathrm{Fe} / \mathrm{H}]=+0.31 \pm 0.05$. The quoted uncertainties do not include systematic errors. For this nearby binary star, $T_{\text {eff }}$ and $\log g$ can be determined by direct methods; i.e., methods are only weakly dependent on models. The angular diameter has been measured by Kervella et al. (2003). Using the updated parallax from van Leeuwen (2007), we determine the radius $R=0.864 \pm 0.005 R_{\odot}$. The mass has been determined from the binary orbit by Pourbaix et al. (2002): $M=0.934 \pm 0.006 M_{\odot}$. Coincidentally, this mass is nearly identical to that of CoRoT7 (LRS09). Combing the mass and radius $\left(g \propto M / R^{2}\right)$ gives a very accurate value of the surface gravity for $\alpha$ Cen B: $\log g=$ $4.538 \pm 0.008$. This is in very good agreement with our spectroscopic determination. As for CoRoT-7, Mg Ib is not useful for constraining $\log g$. The $T_{\text {eff }}$ can be determined from the angular diameter and the bolometric flux: $T_{\mathrm{eff}}=5140 \pm 56$ (Bruntt et al. 2010), agreeing with the result from VWA. Porto de Mello et al. (2008) listed the results of 14 different analyses of $\alpha$ Cen B, based on different methods and quality of the data. Our value of $T_{\text {eff }}$ is in good agreement with previous determinations but our metallicity is slightly higher than most previous estimates.

To conclude, our analysis of the spectra of the Sun and $\alpha$ Cen B show that we can reliably determine $T_{\text {eff }}$ and $\log g$. Since these two stars bracket CoRoT-7 in terms of spectral type, we have confidence that the spectroscopic results are robust and do not suffer from significant systematic errors. We discuss the uncertainties on the spectroscopic parameters in Sect. 6.1. 


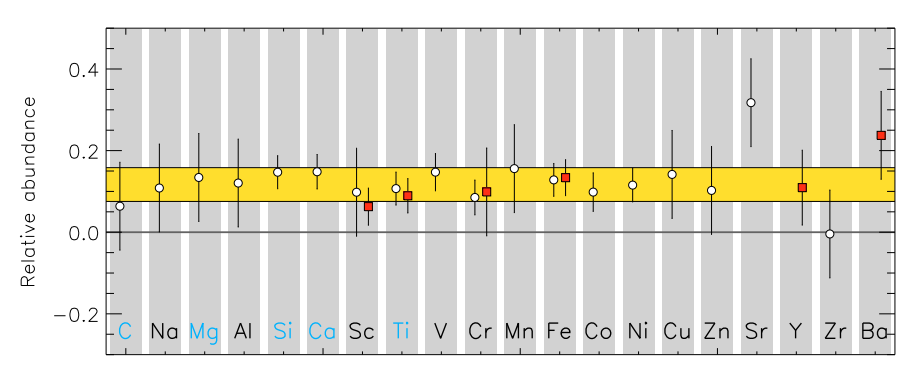

Fig. 5. Mean abundances of 20 elements in CoRoT-7 determined from the H1-107 spectrum. Circle and box symbols are used for neutral and singly ionised lines, respectively. The horizontal bar indicates the mean metallicity and the $1-\sigma$ error range, $[\mathrm{M} / \mathrm{H}]=0.12 \pm 0.04$. The horizontal line at 0.0 corresponds to the solar abundance.

Table 4. Abundances relative to the Sun for 20 elements in CoRoT-7, with the number of lines used for each element.

\begin{tabular}{|c|c|c|c|c|c|}
\hline$\overline{\mathrm{CI}}$ & +0.06 & 1 & Mn I & +0.16 & $\overline{2}$ \\
\hline $\mathrm{Na} \mathrm{I}$ & +0.11 & 1 & $\mathrm{Fe} \mathrm{I}$ & $+0.13 \pm 0.04$ & 143 \\
\hline Mg I & +0.13 & 1 & $\mathrm{Fe}$ II & $+0.13 \pm 0.04$ & 16 \\
\hline $\mathrm{Al} \mathrm{I}$ & +0.12 & 2 & Co I & $+0.10 \pm 0.05$ & 6 \\
\hline Si I & $+0.15 \pm 0.04$ & 6 & $\mathrm{Ni}$ I & $+0.12 \pm 0.04$ & 40 \\
\hline $\mathrm{Ca} \mathrm{I}$ & $+0.15 \pm 0.04$ & 7 & $\mathrm{Cu} \mathrm{I}$ & +0.14 & 1 \\
\hline $\mathrm{Sc} \mathrm{I}$ & +0.10 & 1 & $\mathrm{Zn} \mathrm{I}$ & +0.10 & 1 \\
\hline Sc II & $+0.06 \pm 0.05$ & 3 & Sr I & +0.32 & 1 \\
\hline Ti I & $+0.11 \pm 0.04$ & 37 & Y II & $+0.11 \pm 0.09$ & 3 \\
\hline Ti II & $+0.09 \pm 0.04$ & 8 & $\mathrm{Zr} \mathrm{I}$ & -0.00 & 2 \\
\hline V I & $+0.15 \pm 0.05$ & 3 & Ba II & +0.24 & 1 \\
\hline Cr I & $+0.09 \pm 0.04$ & 8 & & & \\
\hline Cr II & $+0.10 \pm 0.04$ & 2 & & & \\
\hline
\end{tabular}

\subsection{The chemical composition of CoRoT-7}

The abundance pattern of CoRoT-7 relative to the Sun is shown in Fig. 5 for the H1-107 spectrum, and in Table 4 we list the individual abundances of 20 elements. We adopted this spectrum since it has the highest $S / N$, but we note that the other spectra give very similar results. The mean metallicity is computed from the mean of the metal abundances for species with at least 30 lines in the spectrum: $\mathrm{Ti}, \mathrm{Fe}$, and $\mathrm{Ni}$. The mean value is $[\mathrm{M} / \mathrm{H}]=+0.12 \pm 0.04$, where the uncertainty includes the uncertainty on $T_{\text {eff }}, \log g$, and $v_{\text {micro }}$. It can be seen that all elements agree with a scaling of +0.12 dex relative to the solar abundance. For elements with a few lines available $(n<3)$, we assume an uncertainty of 0.1 dex.

\subsection{Determination of $v \sin i$ and $v_{\text {macro }}$}

From the detailed profile shapes of isolated lines, one can ultimately extract information about the granulation velocity fields (Dravins 2008). However, this is impossible with our data where each single spectrum only has $S / N \approx 60$. The intrinsic shape of a spectral line is determined by several factors (Gray 2008), but the broadening due to stellar rotation and velocity fields in the atmosphere can be described to a good approximation by two parameters: $v \sin i$ and macroturbulence ( $\left.v_{\text {macro }}\right)$. These two parameters describe the projected velocity field due to rotation of a limb-darkened sphere and the movement of granules due to convection, respectively.

To measure $v \sin i$ and $v_{\text {macro }}$, we selected 64 isolated lines of different metal species: $\mathrm{Ni}, \mathrm{Ca}, \mathrm{Ti}, \mathrm{Cr}$, and $\mathrm{Fe}$. The lines lie in the range 5800-6450 $\AA$ with equivalent widths from $25-125 \mathrm{~m} \AA$. For each line we determine the small wavelength shifts needed

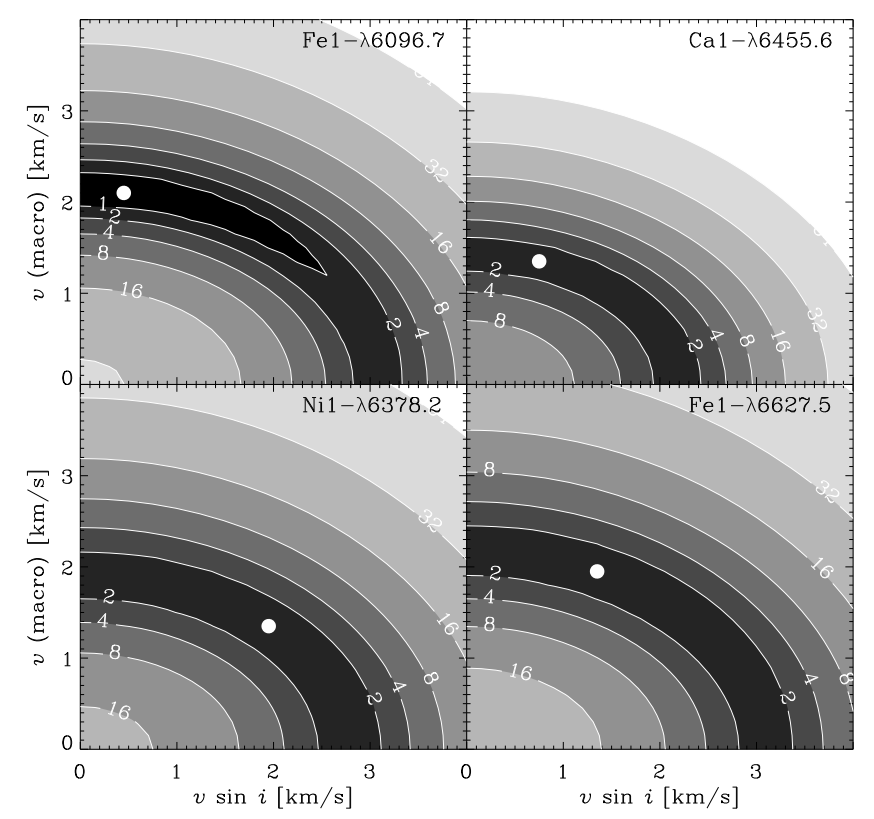

Fig. 6. Contours showing the reduced $\chi^{2}$ values computed for four lines. The synthetic profiles have been convolved with different combinations of $v \sin i$ and $v_{\text {macro }}$. The minimum of the surface is marked by a circle.

so the observed line core fits the synthetic spectrum. This was done by fitting a Gaussian to the line cores of the observed and synthetic spectra. We then fitted the abundance of the line for the adopted $T_{\text {eff }}, \log g$, and $v_{\text {micro }}$. We made a grid of values for $v \sin i$ and $v_{\text {macro }}$ from $0-6 \mathrm{~km} \mathrm{~s}^{-1}$ with steps of $0.15 \mathrm{~km} \mathrm{~s}^{-1}$. For each grid point, we convolved the synthetic spectrum and computed the reduced $\chi^{2}$ of the fit to the observed line. In Fig. 6 we show examples of the $\chi^{2}$ contours for four fitted lines. The generally low reduced $\chi^{2}$ values indicate that our simple representation of the line broadening is successful. It can be seen that there is a strong correlation between the two parameters. The typical $v_{\text {macro }}$ value for a G9V star is about $1-2 \mathrm{~km} \mathrm{~s}^{-1}$ (Gray 2008). For this range the $v \sin i$ values for $\chi^{2}<2$ is below $2.5 \mathrm{~km} \mathrm{~s}^{-1}$ for nearly all lines. From this analysis we find mean values of $v \sin i=1.1_{-0.5}^{+1.0} \mathrm{~km} \mathrm{~s}^{-1}$ and $v_{\text {macro }}=1.2_{-0.5}^{+1.0} \mathrm{~km} \mathrm{~s}^{-1}$. From the analysis of the contours, as shown in Fig. 6 , we can place a firm upper limit of $v \sin i<3 \mathrm{~km} \mathrm{~s}^{-1}$.

From the transit light curve, LRS09 have constrained the inclination angle to be $i=80.1 \pm 0.3^{\circ}$ (see their Fig. 19). Thus, the equatorial rotational velocity is $v_{\text {rot }} \approx v \sin i=1.1_{-0.5}^{+1.0} \mathrm{~km} \mathrm{~s}^{-1}$. This result is only valid if we assume that the inclination of the rotation axis of the star is the same as the inclination of the orbit. LRS09 propose that the rotation period is 23 days $^{2}$, based on the variation in the CoRoT light curve. Using the radius determined in Sect. 6, we get $v_{\text {rot }}=1.7 \pm 0.2 \mathrm{~km} \mathrm{~s}^{-1}$, in agreement with value determined from the spectroscopy.

In Table 2 we list the mean values of $v \sin i$ and $v_{\text {macro }}$ that we have determined for several of the spectra. We did not use the U1 spectrum since it has a lower resolution than the HARPS spectra. We also did not consider the H1-107 spectrum since it is a combination of so many spectra, which inevitably leads to less well-defined line shapes.

\footnotetext{
${ }^{2}$ We adopt an uncertainty on the rotation period of 2 days.
} 


\section{Spectroscopy made easy (SME)}

In an independent analysis of the $\mathrm{H} 1$ and $\mathrm{H} 1-107$ spectra, we used the SME package (version 2.1, Valenti \& Piskunov 1996; Valenti \& Fischer 2005). This code uses a grid of stellar models (Kurucz models or MARCS models) to iteratively determine the fundamental stellar parameters. This is done by fitting the observed spectrum to a synthesised spectrum and minimising the discrepancies through a non-linear least-squares algorithm. SME is based on the philosophy (Valenti \& Piskunov 1996) that, by matching synthetic spectra to observed line profiles, one can extract the information in the observed spectrum and search among stellar and atomic parameters until the best fit is achieved.

We used a large number of spectral lines, e.g. the Balmer lines (the extended wings are used to constrain $T_{\text {eff }}$ ), NaID, $\mathrm{Mg}$ I b, and Ca I (for $T_{\text {eff }}$ and $\log g$ ) and metal lines (to constrain the abundances). Furthermore, the iterative fitting provided information on micro- and macroturbulence and $v \sin i$.

By fitting the extended wings of the $\mathrm{H} \alpha$ and $\mathrm{H} \beta$ Balmer lines, we determined the $T_{\text {eff }}$ to be $5200 \mathrm{~K}$ and $5100 \mathrm{~K}$ respectively. Using instead the Na I doublet at $\lambda 5890 \AA$, we found a $T_{\text {eff }}$ of $5280 \mathrm{~K}$. The lower value derived from the $\mathrm{H} \beta$ line wings is explained by the many metal lines contributing to the profile. We tried to use the $\mathrm{Mg} I \mathrm{~b}$ triplet to evaluate $\log g$, but as for the VWA analysis, we find that it is difficult to assign the continuum level, so instead we used the wide Ca I lines. From the SME analysis, we find the $\log g$ to be 4.43 from $\mathrm{Mg}$ I and 4.49 from Ca I. Our evaluation of the metallicity is $[\mathrm{M} / \mathrm{H}]=+0.13$ and $v_{\text {micro }}=0.80 \mathrm{~km} \mathrm{~s}^{-1}$.

The uncertainties using SME determined by Valenti \& Fischer (2005), found from a large sample of more than 1000 stars, are $44 \mathrm{~K}$ in $T_{\text {eff }}, 0.06$ dex in $\log g$, and 0.03 dex in $[\mathrm{M} / \mathrm{H}]$, which we adopt for our SME analysis of CoRoT-7. In a few cases, Valenti \& Fischer (2005) find offsets of up to 0.3 dex for $\log g$. When we compared the results for CoRoT-7 for different lines and methods used to constrain $\log g$, we found a scatter of 0.06 dex. This is consistent with the results of Valenti \& Fischer (2005), and we assign this as the 1- $\sigma$ uncertainty.

In summary, the parameters determined with SME for the H1 and H1-107 spectra of CoRoT-7 give fully consistent results with the more extensive analysis with VWA. Our results from the SME analysis are given in Table 2.

\section{Absolute magnitude from the Wilson-Bappu effect}

The width of the emission peaks seen in the core of the Ca II H \& K lines (3934.8 and $3969.7 \AA$ ) in late-type stars are directly correlated to the value of $\log g$, and hence to the mass and radius of the star. This implies that the width can be calibrated in terms of the absolute luminosity (Gray 2008). The calibration of the absolute magnitude is of the form: $M_{V}=a \log W_{0}+b$, where $W_{0}$ is the width at the zero-level of the emission component, and where the constants $a$ and $b$ also need to be properly calibrated. This is usually done using data from clusters, and we have used the recent calibration of Pace et al. (2003), who found $a=-18.0$ and $b=33.2$ with a quoted uncertainty of $0.6 \mathrm{mag}$ on $M_{V}$.

In Fig. 7, we show the Ca II H \& K lines of CoRoT-7. The emission components with self-reversal in the line cores are clearly seen. By measuring the width of both the $\mathrm{H}$ - and the K-lines, following the method of Pace et al. (2003), we find an absolute magnitude of $M_{V}=5.4 \pm 0.6$. Given the spectroscopic effective temperature, the location in the Hertzsprung-Russell diagram indicates that CoRoT-7 is a main sequence star with

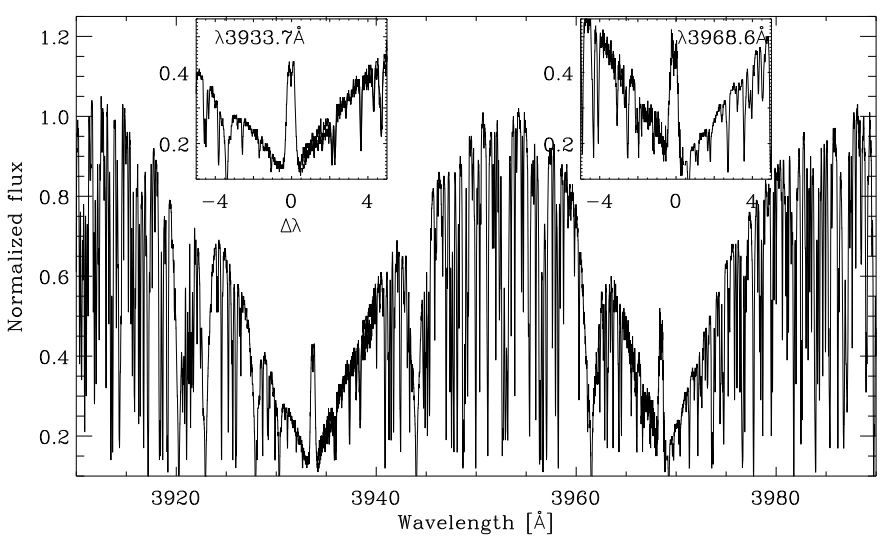

Fig. 7. The emission component of the Ca II H\& K line of CoRoT-7. The self-reversal in the emission cores is shown in the insets.

Table 5. Parameters of CoRoT-7. Parameters from isochrones/tracks use the age limit $A<2.3$ Gyr from LRS09.

\begin{tabular}{lrll}
\hline \hline Parameter & \multicolumn{1}{c}{ Value } & Unit & Method \\
\hline$T_{\text {eff }}$ & $5250 \pm 60$ & $\mathrm{~K}$ & Spectroscopy \\
$\log g$ & $4.47 \pm 0.05$ & & Spectroscopy \\
{$[\mathrm{Fe} / \mathrm{H}]$} & $+0.12 \pm 0.06$ & & Spectroscopy \\
$L / M$ & $0.62 \pm 0.08$ & $L_{\odot} / M_{\odot}$ & Spectr.: $L / M \propto T_{\mathrm{eff}}^{4} / g$ \\
\hline$M_{\star}$ & $0.91 \pm 0.03$ & $M_{\odot}$ & Isochrones/tracks \\
$R_{\star}$ & $0.82 \pm 0.04$ & $R_{\odot}$ & Isochrones/tracks \\
$L_{\star}$ & $0.49 \pm 0.07$ & $L_{\odot}$ & Isochrones/tracks \\
$\log g$ & $4.57 \pm 0.05$ & & Isochrones/tracks \\
\hline
\end{tabular}

spectral type in the range is $\mathrm{G} 8 \mathrm{~V}-\mathrm{K} 0 \mathrm{~V}$. That the star is not evolved is in good agreement with the $\log g$ determination.

\section{Evolutionary status}

We now evaluate the atmospheric parameters determined above for CoRoT-7 and compare with evolutionary models to constrain the mass, radius, and luminosity.

\subsection{Final atmospheric parameters of CoRoT-7}

There is generally good agreement for determination of $T_{\text {eff }}$ using VWA and SME. With the VWA method we only used Fe I-II lines, while with SME we also used the Balmer lines to constrain $T_{\text {eff }}$. As mentioned, the quoted uncertainties in Table 2 only include the intrinsic error of the method, i.e. by varying the model parameters. However, the temperature and pressure profile of the atmospheric model may not fully represent the actual star. From the analysis of the Sun and $\alpha$ Cen B, we found good agreement for their $T_{\text {eff }}$ and $\log g$ determined from modelindependent methods (see Sect. 3.4). Thus, there appears to be no large systematic errors. Bruntt et al. (2010) have analysed a larger sample of stars, comparing the spectroscopic $T_{\text {eff }} \mathrm{S}$ with those from fundamental methods (as done for $\alpha$ Cen B here) and find a systematic offset in $T_{\text {eff }}$ of $-40 \pm 20 \mathrm{~K}$. We included this offset to get the final value $T_{\text {eff }}=5250 \pm 60 \mathrm{~K}$. We used several pressure-sensitive spectral features to constrain $\log g$, and the mean value we adopt is $\log g=4.47 \pm 0.05$. For $T_{\text {eff }}$ and $\log g$, we added systematic errors on $50 \mathrm{~K}$ and 0.05 dex, based on the discussion by Bruntt et al. (2010). The mean metallicity is found to be $[\mathrm{M} / \mathrm{H}]=+0.12 \pm 0.06$, where we increased the uncertainty because we included of systematic errors on $T_{\mathrm{eff}}$ and 


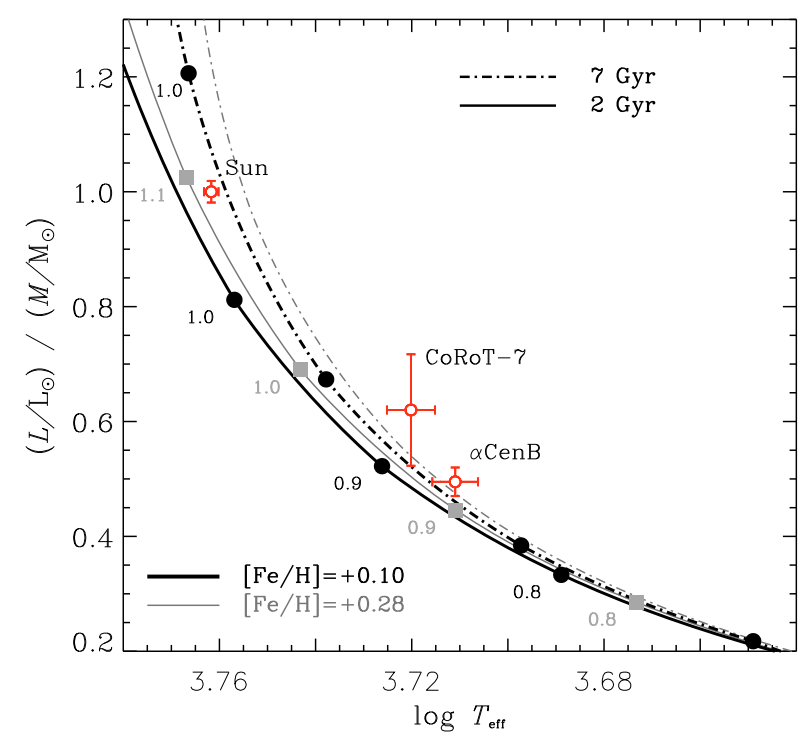

Fig. 8. BASTI isochrones with different ages and metallicities. Filled circles and boxes mark selected mass points. The determined $L / M$ ratios for CoRoT-7, $\alpha$ Cen B, and the Sun are plotted as open symbols.

$\log g$. These are our final estimates for the parameters of CoRoT7 as summarised in Table 5.

Our new results for the fundamental parameters are in good agreement with LRS09. They find $T_{\text {eff }}=5275 \pm 75 \mathrm{~K}$ as a mean value of different groups using different spectroscopic analyses of the UVES spectrum. They also use a calibration with 2MASS infrared photometry, taking interstellar reddening into account, yielding $5300 \pm 70 \mathrm{~K}$. They find $\log g=4.50 \pm 0.10$ using the Fe I-II equilibrium criterion and the $\mathrm{Mg}$ I b and $\mathrm{Na}$ I D lines, which is also in good agreement with our value. LRS09 found a slightly lower metallicity, $[\mathrm{M} / \mathrm{H}]=+0.03 \pm 0.06$ (our revised value for the same spectrum is $[\mathrm{M} / \mathrm{H}]=+0.11 \pm 0.06)$. Several strong lines were included in that analysis, while in this study we only use Fe I lines with $E W<90 \mathrm{~m} \AA$. For other elements (and Fe II), we included lines with $E W<140 \mathrm{~m} \AA$. This choice was made because the strong lines start to be saturated and are therefore less sensitive to changes in the atmospheric parameters. For comparison, $250 \mathrm{Fe}$ I and $18 \mathrm{Fe}$ II lines were used by LRS09 while we used only 143 and 16, respectively. In our analysis we used Fe lines in the wavelength range 4880-6865 $\AA$, while LRS09 included several lines in the blue region (4515-6865 $\AA$ ). The current version of VWA does not take molecular lines into account, which start to become a problem for such a cool star, especially at short wavelengths.

\subsection{Stellar mass, luminosity, and radius}

In some cases the modelling of the transit light curve can be used to obtain the mean density of the star. However, as pointed out by LRS09, the shallow eclipse combined with stellar activity modulating the light curve seriously hampers such analyses. From the spectroscopic value of $\log g$ we have an estimate of $g=G M / R^{2}$. Multiplying this with the relation $L \propto R^{2} T_{\mathrm{eff}}^{4}$, we can eliminate the radius, i.e. $L / M \propto T_{\mathrm{eff}}^{4} / g$. Thus, we determine the luminosity-mass ratio: $\left(L / L_{\odot}\right) /\left(M / M_{\odot}\right)=0.62 \pm 0.08$. The uncertainty is dominated by the uncertainty on the surface gravity.

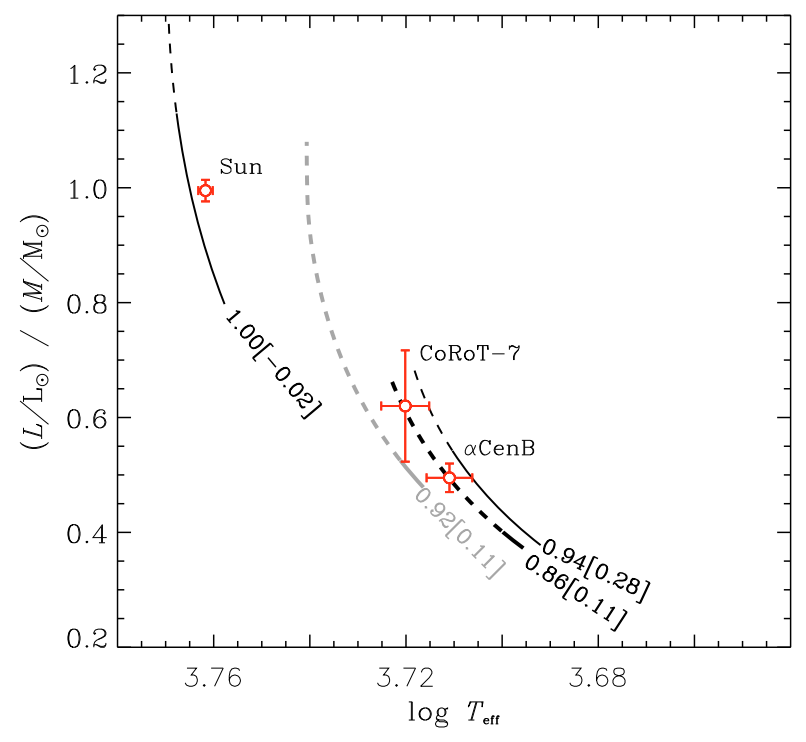

Fig. 9. Four ASTEC evolution tracks for different masses and metallicities. A track for $1.00 M_{\odot}$ and $[\mathrm{Fe} / \mathrm{H}]=-0.02$ is shown near the Sun. The determined $L / M$ ratios for CoRoT-7, $\alpha$ Cen B, and the Sun are plotted as open symbols. Dashed lines are used for ages higher than the adopted limits on the age, i.e. 4.6 Gyr for the Sun, 2.3 Gyr for CoRoT-7, and $6.5 \mathrm{Gyr}$ for $\alpha \mathrm{Cen} \mathrm{B}$, while the maximum possible age is $14 \mathrm{Gyr}$.

In Figs. 8 and 9, we compare this estimate with isochrones from BASTI (Pietrinferni et al. 2004) and evolution tracks from ASTEC (Christensen-Dalsgaard 2008). These models do not include overshoot, but this has no impact on low-mass stars such as CoRoT-7. The mixing-length parameter for the ASTEC grid is $\alpha_{\mathrm{ML}}=1.8$. The models express metallicity in terms of the heavy element mass fraction, $Z$. To convert each $Z$ to spectroscopic values, we adopt the solar value $Z_{\odot}=0.0156$ (Caffau et al. 2009) with an assumed uncertainty of 0.002 . This corresponds to an increase in the uncertainty of $[\mathrm{Fe} / \mathrm{H}]$ by $0.05 \mathrm{dex}$.

In Fig. 8 we show two sets of isochrones with metallicity $[\mathrm{Fe} / \mathrm{H}]=+0.10$ and +0.28 for ages of 2 and $7 \mathrm{Gyr}$, with several mass points indicated in the range 0.8 to $1.1 M_{\odot}$. The lower metallicity is close to that of CoRoT-7, and the higher metallicity represents $\alpha$ Cen B. The uncertainty on $L / M$ for CoRoT-7 is relatively large, so we cannot constrain the mass without further constraints. Fortunately, LRS09 estimate the age of CoRoT7 from the rotation period and the activity index of the $\mathrm{Ca} \mathrm{H} \& \mathrm{~K}$ lines: 1.2-2.3 Gyr. By adopting this age limit, we can estimate the mass and radius from the isochrones: $M / M_{\odot}=0.89 \pm 0.03$ and $R / R_{\odot}=0.80 \pm 0.04$.

In Fig. 9 we show four selected ASTEC evolution tracks that represent the Sun, CoRoT-7 (two tracks), and $\alpha$ Cen B. The dashed part of each track is for ages above these adopted limits: 4.6 Gyr for the Sun (1.00 $M_{\odot}$ track), 2.3 Gyr for CoRoT-7 (0.92 and $\left.0.86 M_{\odot}\right)$, and $6.5 \mathrm{Gyr}$ for $\alpha$ Cen B $\left(0.94 M_{\odot}\right.$, see Miglio \& Montalbán 2005, for discussion of the age of $\alpha$ Cen A+B). Furthermore, the tracks all end at 14 Gyr. It is seen that the Sun is quite well represented, although the $L / M$ ratio is quite high at $4.6 \mathrm{Gyr}$, but this is explained by the available track having slightly too low metallicity. The $0.94 M_{\odot}$ track for $\alpha$ Cen B agrees with $L / M$ within the $1-\sigma$ limit. For CoRoT-7, the $0.86 M_{\odot}$ track does not reach the determined $T_{\text {eff }}$ and $L / M$ in $2.3 \mathrm{Gyr}$. However, the $0.92 M_{\odot}$ track agrees with the $T_{\text {eff }}$ and $L / M$. From similar tracks we determine these limits on the mass and radius 
of CoRoT-7: $M / M_{\odot}=0.92 \pm 0.03$ and $R / R_{\odot}=0.83 \pm 0.04$. This is in good agreement with the result from the BASTI tracks. As our final result, we adopt the mean radius and mass determined from the two sets of models: $M / M_{\odot}=0.91 \pm 0.03$ and $R / R_{\odot}=0.82 \pm 0.04$.

LRS09 determined slightly different values for the mass and radius. They used the STAREVOL evolution tracks (Palacios, priv. comm.) with slightly different stellar atmospheric values. Their values are $M / M_{\odot}=0.93 \pm 0.03$ and $R / R_{\odot}=0.87 \pm 0.04$ (i.e. $\log g=4.53 \pm 0.04$ ), which agree quite well with our revised results given in Table 5 .

From comparison with the BASTI and ASTEC models the determined $L / M$ of CoRoT-7 seems to be too high, although the uncertainty is large. To determine the luminosity, we therefore adjust the ratio by $-1 \sigma,\left(L / L_{\odot}\right) /\left(M / M_{\odot}\right)=0.54 \pm 0.08$, and multiply by the inferred mass to get $L / L_{\odot}=0.49 \pm 0.07$. The determined mass and radius from the isochrones correspond to a surface gravity $\log g=4.57 \pm 0.04$, which is slightly higher $(1.6 \sigma)$ than the spectroscopic value of $4.47 \pm 0.05$.

To confirm that the BASTI and ASTEC models can be used for CoRoT-7 we also plot the Sun and $\alpha$ Cen B in Figs. 8 and 9. For $\alpha$ Cen B the uncertainty is much less than for CoRoT-7: $\left(L / L_{\odot}\right) /\left(M / M_{\odot}\right)=0.50 \pm 0.02$. Miglio \& Montalbán (2005) determined an age of about $6.5 \mathrm{Gyr}$ for the $\alpha$ Cen $\mathrm{A}+\mathrm{B}$ system, and with our metallicity of +0.3 dex, there is good agreement with both sets of models. From comparison with the BASTI and ASTEC models, we get the mass $0.90 \pm 0.03 M_{\odot}$, which agrees well with the dynamical mass of $0.934 \pm 0.006 M_{\odot}$. The radius is $0.84 \pm 0.04 R_{\odot}$, where the interferometric result is $R=$ $0.864 \pm 0.005 R_{\odot}$. Combining the mass and radius from the comparison with the isochrones, we get $\log g=4.54 \pm 0.04$, which is in good agreement with the spectroscopic value of $4.50 \pm 0.03$.

\section{Discussion and conclusion}

We have presented a detailed spectroscopic analysis of the planet-hosting star CoRoT-7. The analysis is based on HARPS spectra, that have higher $S / N$ and better resolution than the UVES spectrum used to get a preliminary result (LRS09). We analysed both individual spectra from different nights and coadded spectra, and found excellent agreement. Only for one of the single HARPS spectra did we find a systematic error in $T_{\text {eff }}$ and $\log g$, which is explained by the low $S / N$.

We described in detail the VWA tool used to determine of the atmospheric parameters $T_{\text {eff }}$ and $\log g$ with Fe I-II lines and the pressure-sensitive $\mathrm{MgI}$ I and $\mathrm{Ca}$ lines. We used the SME tool to also analyse the Balmer and $\mathrm{Na}$ I lines. We find excellent agreement between the different methods.

To evaluate the evolutionary status (age) and fundamental stellar parameters (mass, radius) we compared the observed properties of CoRoT-7 with theoretical isochrones. From the spectroscopic $T_{\text {eff }}$ and $\log g$ we can estimate $L / M$. We compared this with isochrones but find that the uncertainty is too large to constrain the evolutionary status. However, by imposing constraints on the stellar age (1.2-2.3 Gyr from LRS09), we can constrain the mass and radius to $0.91 \pm 0.03 M_{\odot}$ and $0.82 \pm 0.04 R_{\odot}$. This is a only slight revision of the original value from LRS09, who used a lower metallicity. The relatively large uncertainty of $7 \%$ on the stellar radius directly affects the accuracy of the determined radius and density of the transiting planet, CoRoT-7b.

We used the new stellar parameters to fit the transit light curve reported by LRS09. We used the formalism of Giménez (2006) with fixed limb-darkening coefficients, and we explored the parameter space, which is consistent with the stellar parameters and their associated uncertainty. The constraints to the fit include the orbital inclination $\left(81.45 \pm 1.10^{\circ}\right)$, the phase of transit ingress $\theta_{1}(0.02785 \pm 0.00005)$, and the ratio of planet-to-star radius $(0.0176 \pm 0.0003)$. We refer to Sect. 9 in LRS09 for a description of the methodology of the fitting procedure. With the new stellar parameters, we determined the radius of the planet to be slightly smaller with radius $1.58 \pm 0.10 R_{\oplus}$ (LRS09 found $\left.1.68 \pm 0.09 R_{\oplus}\right)$. The slightly smaller radius is mainly due to our revision of the stellar radius.

The new stellar mass and the updated inclination were used with the published values of the ephemeris (LRS09) and radial velocity semiamplitude (Queloz et al. 2009) to estimate the mass of the planet CoRoT-7b as $5.2 \pm 0.8 \mathrm{M}_{\oplus}$. Combined with the radius of the planet, this results in a density of $7.2 \pm 1.8 \mathrm{~g} \mathrm{~cm}^{-3}$, which is consistent, but slightly denser than the reported value of $5.6 \pm 1.3 \mathrm{~g} \mathrm{~cm}^{-3}$ in the previous work.

We also analysed spectra of the Sun and $\alpha$ Cen B, observed with the HARPS spectrograph. For these stars the fundamental parameters are known with very good accuracy, so they can be used to validate our methods for the much fainter star CoRoT-7. We compared the spectroscopically determined $T_{\text {eff }}$ and $\log g$ with the values from fundamental methods for $\alpha$ Cen B, i.e. using the binary dynamical mass and the interferometric determination of the radius. There is excellent agreement within $1-\sigma$, indicating that the adopted uncertainties are realistic. This gives us some confidence that we can use theoretical evolution models to constrain the radii and masses of stars, but requires that limits be put on the stellar age.

The exoplanet host star CoRoT-7 is a slowly rotating, metal rich, type G9V star. The star is relatively faint, and its fundamental parameters can only be determined through indirect methods. The expected future discoveries of similar planet systems with CoRoT and Kepler, will also be limited by our ability to characterise the host stars. In the case of Kepler we have the additional advantage, that for the brightest stars, the solar-like pulsations can be used to constrain the stellar radius (Christensen-Dalsgaard et al. 2010). This analysis also relies on evolution models but will be able to constrain the stellar radius to about 2\% (Stello et al. 2009; Basu et al. 2010). For most of the Kepler targets, astrometric parallaxes will be available, while for CoRoT-7 we must wait for the GAIA mission.

Acknowledgements. We are thankful to Nikolai Piskunov (Uppsala Astronomical Observatory) for making SME available to us and for answering numerous questions. We are grateful for the availability of the VALD database for the atomic parameters used in this work. The spectra we used are from observations made with ESO Telescopes at the La Silla and Paranal Observatories under programme IDs 081.C-0413(C), 082.C-0120, 082.C-0308(A), 282.C-5036(A), and 60.A-9036(A).

\section{References}

Baglin, A., Michel, E., Auvergne, M., \& The COROT Team. 2006, in Beyond the spherical Sun ,ESA Special Publication, 624, Proceedings of SOHO 18/GONG 2006/HELAS I

Barklem, P. S., Piskunov, N., \& O’Mara, B. J. 2000, A\&AS, 142, 467

Basu, S., Chaplin, W. J., \& Elsworth, Y. 2010, ApJ, 710, 1596

Bruntt, H. 2009, A\&A, 506, 235

Bruntt, H., Catala, C., Garrido, R., et al. 2002, A\&A, 389, 345

Bruntt, H., Bikmaev, I. F., Catala, C., et al. 2004, A\&A, 425, 683

Bruntt, H., De Cat, P., \& Aerts, C. 2008, A\&A, 478, 487

Bruntt, H., Bedding, T. R., Quirion, P., et al. 2010, MNRAS, 746

Caffau, E., Maiorca, E., Bonifacio, P., et al. 2009, A\&A, 498, 877

Christensen-Dalsgaard, J. 2008, Ap\&SS, 316, 13

Christensen-Dalsgaard, J., Kjeldsen, H., Brown, T. M., et al. 2010, ApJ, 713, L164 
A\&A 519, A51 (2010)

Cox, A. N. 2000, Allen's astrophysical quantities (New York: AIP Press, Springer)

Deleuil, M., Deeg, H. J., Alonso, R., et al. 2008, A\&A, 491, 889

Dravins, D. 2008, A\&A, 492, 199

Fridlund, M., Hébrard, G., Alonso, R., et al. 2010, A\&A, 512, A14

Fuhrmann, K., Pfeiffer, M., Frank, C., Reetz, J., \& Gehren, T. 1997, A\&A, 323, 909

Giménez, A. 2006, A\&A, 450, 1231

Gray, D. F. 2008, The Observation and Analysis of Stellar Photospheres (Cambridge University Press)

Gustafsson, B., Edvardsson, B., Eriksson, K., et al. 2008, A\&A, 486, 951

Heiter, U., Kupka, F., van't Veer-Menneret, C., et al. 2002, A\&A, 392, 619

Hinkle, K., Wallace, L., Valenti, J., \& Harmer, D. 2000, Visible and Near Infrared Atlas of the Arcturus Spectrum 3727-9300 A (San Francisco: ASP)

Kervella, P., Thévenin, F., Ségransan, D., et al. 2003, A\&A, 404, 1087

Kupka, F., Piskunov, N., Ryabchikova, T. A., Stempels, H. C., \& Weiss, W. W. 1999, A\&AS, 138, 119
Kurucz, R. L., Furenlid, I., Brault, J., \& Testerman, L. 1984, Solar flux atlas from 296 to $1300 \mathrm{~nm}$ (New Mexico, USA: National Solar Observatory, Sunspot)

Léger, R., Schneider, B. P., Fridlund, M., et al. 2009, A\&A, 506, 287

Mayor, M., Pepe, F., Queloz, D., et al. 2003, The Messenger, 114, 20 Miglio, A., \& Montalbán, J. 2005, A\&A, 441, 615

Moutou, C., Bruntt, H., Guillot, T., et al. 2008, A\&A, 488, L47

Pace, G., Pasquini, L., \& Ortolani, S. 2003, A\&A, 401, 997

Pietrinferni, A., Cassisi, S., Salaris, M., \& Castelli, F. 2004, ApJ, 612, 168

Porto de Mello, G. F., Lyra, W., \& Keller, G. R. 2008, A\&A, 488, 653

Pourbaix, D., Nidever, D., McCarthy, C., et al. 2002, A\&A, 386, 280

Queloz, D., Bouchy, F., Moutou, C., et al. 2009, A\&A, 506, 303

Rauer, H., Queloz, D., Csizmadia, S., et al. 2009, A\&A, 506, 281

Stello, D., Chaplin, W. J., Bruntt, H., et al. 2009, ApJ, 700, 1589

Valenti, J. A., \& Fischer, D. A. 2005, ApJS, 159, 141

Valenti, J. A., \& Piskunov, N. 1996, A\&AS, 118, 595

van Leeuwen, F. 2007, A\&A, 474, 653 